\title{
Learning Information Diffusion Models from Observation and Its Application to Behavior Analysis
}

\author{
Hiroshi Motoda \\ The Institute of Scientific and Industrial Research \\ Osaka University, Japan \\ motoda@ar.sanken.osaka-u.ac.jp
}

\begin{abstract}
We investigate how well different information diffusion models can explain observation data by learning their parameters and discuss which model is more appropriate to which topic. We use two models, one from push type diffusion (AsIC) and the other from pull type diffusion (AsLT), both of which are extended versions of the well known Independent Cascade (IC) and the Linear Threshold (LT) models that incorporate asynchronous time delay. The model parameters are learned by maximizing the likelihood of the observed data being generated by an EM like iterative search, and the model selection is performed by choosing the one with better predictive power. We first show by using four real networks that the proposed learning algorithm correctly learns the model parameters both accurately and stably, and the proposed selection method identifies the correct diffusion model from which the data are generated. We then apply these methods to behavioral analysis of topic propagation using a real blog diffusion sequence, and show that although the inferred relative diffusion speed and range for each topic is rather insensitive to the model selected, there is a clear indication of which topic to better follow which model. The correspondence between the topic and the model selected is indeed interpretable.
\end{abstract}

